\title{
Communication in Medicine
}

\section{Communication in medical practice across ethnic boundaries}

\author{
A.H. Crisp and W.J. Edwards* \\ St George's Hospital Medical School, Cranmer Terrace, London SWI7 0RE, UK.
}

\begin{abstract}
Summary: The communication skills basic to good medical practice are, for many of us, unnatural. The authors attempt to address aspects of the matter with particular reference to communication across ethnic boundaries.
\end{abstract}

The aim of medical practice is to relieve suffering and symptoms, to cure and to promote and maintain health. This requires a problem-solving approach to the human condition in both physical and social frameworks.

The Flexner approach to medical practice has led to great advances in medicine at the microbiological and technical levels but with less attention being paid to social and psychological factors within health and disease. In particular, it has sometimes therefore failed to do justice to the importance of these factors in the clinical consultation, where communication is inevitable, and the proper conduct of which is the essential process for accurate diagnosis and intervention.

In addition, medical practice in the West these days often involves a carer and a patient or client who come from different ethnic groups. This has for long been true within North America. It is now commonplace in the European Economic Community (EEC) and in the United Kingdom in particular where it is often highlighted by differences in skin colour.

Everyday communication between people often subserves their personal needs of a protective and self-promotional kind. Moreover, hostility can be expressed by resistance to or avoidance of effective communication. Individuals who enjoy conflict will reflect this through strategically combative, distracting and manipulative communication that blocks solution to basic problems. Barriers to good communication between individuals will thus also reflect their personalities and the nature of their relationships.

Communication aimed at helping someone else to resolve his or her problem is in many senses unnatural in most circumstances and has to be learned by most of

Correspondence: Professor A.H. Crisp, M.D., F.R.C.Psych.

* Present address: St Anne's Psychiatric Hospital, Port of Spain, Trinidad, West Indies

Accepted: 24 October 1988 us. However, such communication is as fundamenta. to good medical practice as it is also to good educations in general. Indeed, the word 'doctor' means 'teacher' $/$ and such skills are therefore essential for both profes-sions.

Within the UK more emphasis has recently been placed on the teaching of communication skills for? medical practice. The Education Committee of the General Medical Council (GMC), which has statutorye responsibility for co-ordinating all stages of medica $\mathrm{B}$ education, has recently issued recommendations which touch on this issue in respect of immedianees postgraduate and further general clinical training a id in respect of subsequent specialist training. In thos connection the former recommendations ${ }^{1}$ state: 'Communication skills: These are fundamental to goodô patient care and include history taking, involvingo patients in decisions about themselves and giving treatment, advice support and counselling. The intro-윽 ductory teaching in the undergraduate curriculum should be reinforced during general clinical training. and the assessment of trainees' performance should take account of their comprehension of cultura 3 values, practices and constraints, and their ability too communicate effectively with patients (including chil dren and the elderly) and patient's families, medicalo colleagues and others involved in patient care.'

The Recommendations on Training of Specialists $\left.{ }^{20}\right]$ identify such skills as one necessary attribute of the doctor stating that possession of consultation skills includes: 'Skills in sensitive and effective communica-No tion with patients and their families, professionat colleagues and local agencies, and the keeping of good medical records.'

This is further elaborated as follows: 'The consult ation is fundamental to clinical medical practice and depends on successful communication between doc- $-\infty$ tors and patients. Good communication requires time. $\cdot$ It also calls for understanding by the doctor of his oro her own temperament. It involves the capacity to take $\mathbb{D}$ a good clinical history, to listen to the patient in a wayd 
that enables the patient to talk openly, and the ability to explain concisely and sensitively, in simple language, the salient features of the patient's illness and any risks or disadvantages inherent in the treatment proposed. It also requires a capacity to assess the patient's understanding of the explanation so that he or she can, where appropriate, decide whether to proceed. Appreciation of the special needs of ethnic minorities is essential to good communication in our multi-racial society. Some aspects of communication may be non-verbal, such as the doctor's manner during the consultation. Communication is also fundamental to the counselling and psychotherapy skills in which doctors in clinical practice may often need to engage, most obviously in specialties such as general practice, psychiatry and obstetrics and gynaecology.'

In now addressing this matter in more detail the authors make the following succession of points:

(a) The goals of training for communication skills in medical practice in general have been identified by various writers. ${ }^{3-7}$ One set of such goals and their objectives and means of achievement are appended (Annexe 1).

(b) Good communication can bring about change, is therefore sometimes experienced as threatening, and may therefore be avoided. On the other hand good communication is the only means by which we can aim to transcend and solve this problem.

(c) The essence of medical practice is its invasiveness. The doctor's training licenses him to invade the body. Such invasiveness can be chemical, surgical, with rays or waves or psychological. Chemical (pharmacological) invasiveness is often reversible, if needs be, as is invasiveness with rays and waves. Surgical invasiveness is not but may often be lifesaving. Psychological invasiveness may not be reversible because of the mind's structure. For instance, if defences against depression, e.g. paranoia or psychogenic pain, are crudely breached, they may remain so and depression may prevail. Psychological interventions based on good communication skills used by doctors are particularly invasive because of the doctor's position. This potentially enables him or her to have special access to the patient's mind and many patients are particularly receptive to the influence of a doctor.

(d) All these matters can be compounded when the transaction crosses an ethnic boundary. ${ }^{8.9}$ Within the present context relevant additional factors may include differing cultures and, relatedly, differing languages. Languages reflect cultures and do not necessarily lend themselves to absolute translation and reduction to common meanings.

(e) Relationships between individuals from different ethnic groups may therefore importantly reflect cultural as well as personal differences and also the special context in which those concerned are meeting. Various wider political and historical factors may be part of the encounter, masking the realities of the individuals concerned. Equally, the individuals concerned can project onto these wider issues their own needs and developmentally derived conflicts. They can thus effectively defend against acknowledging the latter, both to themselves and others, and sometimes also engage in sustained conflict within one-to-one relationships that embody such culturally different backgrounds - the doctor/patient relationship being an example.

(f) It is perfectly possible to communicate well across ethnic boundaries and despite language difficulties if people want to understand and also help each other. For this to happen either one or the other must wish to learn about the other unreservedly or both must be interested in each other and recognize the possibility of change in themselves. When it comes to the ethnic minorities in the United Kingdom the greatest change is likely to be required of the minorities. This requires the majority to be especially sympathetic and empathic.

(g) Within medical practice problems of communication may arise on the basis that either the doctor or the patient comes from an ethnic minority. It most often arises when the doctor comes from an ethnic minority and has recently settled in this country (e.g. in about $20 \%$ of all medical practice). Increasingly, medical students in the United Kingdom come from an ethnic minority background (perhaps $5 \%-10 \%$ of all medical students) and were themselves born and have undergone primary and secondary education in the UK. It also significantly occurs where the patient comes from an ethnic minority, i.e. in about $6 \%$ of practice. If empathy is at the basis of this problem, then we need to understand what is required for it. Empathy is different from over-involvement and also requires attention to transference and countertransference influences. Empathy can only arise through knowledge of the other person and this has to involve their history, genetic and social.

(h) This has implications not only for medical education but also for secondary and rimary education. Implicit in it is social desegregation, the pathway to which is political and long term. Meanwhile, in day to day practice and in the here and now, attention needs to be paid to the principles governing good education in medical practice within the consultation and with the aim of maximizing the potential for problem-solving. Until such times as society as a whole changes, this will require specific training within medicine. This training should come within the framework of 
training in the development of communication skills. The additional training required for the development of these skills across ethnic boundaries is outlined in Annexe 2.

\section{Governance of the training in communication skills}

The role of the Education Committee of the GMC has already been referred to. The Committee has issued Recommendations which touch, as already indicated, on these matters.

In the UK it is the specialist Royal Colleges, Faculties and Joint Higher Training Committees that determine the detailed content of specialist training and that set and maintain standards through a variety of mechanisms including examinations. For the above learning to occur these training bodies would have to include requirements for it as an aspect of their regulations. As the authors state, it is also a matter for primary and secondary education as well as for undergraduate medical education. The former requires societal changes for it to be effective. Undergraduate medical education is directly governed by the universities whose medical curricula are shaped by the GMC's Recommendations on Basic Medical Education (1980). ${ }^{10}$ These make special reference to the importance of communication skills including the need to understand the impact of cultures upon them.

Within the EEC, the Advisory Committee on Medical Training has recently considered the matter within the framework of the training of doctors for the twenty-first century. ${ }^{11}$ It is to be hoped that future EEC directives concerning medical education refer appropriately to such training.

\section{Annexe 1}

\section{Goals and objectives; basic clinical communication skills:}

(Derived from earlier sources ${ }^{5,12}$ ).

Goals:

(1) The ability to recognize the defensive strategies within the mind, which distort and mask past and present stressful experiences and interactions, and the acknowledgement that they operate within oneself as well as others and are related both to personality and the immediate situation.

(2) The ability to acknowledge and understand how such mental mechanisms often importantly operate within us as carers as well as in our patients, and in our various relationships and especially within the doctor/patient relationship.

(3) The ability thereby to modify one's own behaviour so as to permit patients truly to identify and express how they feel and to recall and share optimally and accurately relevant aspects of their background.
(4) As a result of such knowledge and skills, additional other medical knowledge and skills, to be able to takea full history within the clinical situation, incorporating relevant social and psychological information into formulation and diagnosis. Also to communicate wifh the patient regarding these matters and possible interve tions both caringly and effectively, thereby helping patients fully and accurately to register the information given to them.

(5) The ability thus to enable patients, when possible, make their own informed decisions concerning the future welfare whilst respecting any limitation and their legal rights.

(6) The ability to teach patients more about their illnesse $\overrightarrow{9}$, how they can help themselves to recover, to avoid recurrence of illness and to maintain good health inclugs ing, when appropriate, being able to counsel them of engage with them in basic psychotherapy so as promote such developments.

Objectives:

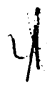

Pursuit and achievement of these goals will concurrentb require and permit optimal progress towards achieving the following specific objectives, so as to enable the trainee, on completion of his training, to conduct an efficient and effective consultation. Thus, the trainee should become able to:

(1) Create the right atmosphere, in terms of professiôn concern and physical ambience.

(2) Sustain a position of personal sensitivity and empathy whilst also being capable of interleaving detachmeg and objective evaluation.

(3) Identify and share with the patient the purposes of the interview.

(4) Listen attentively and receptively, enabling the patien to volunteer personal and other relevant information and to feel involved in his/her own care.

(5) Pursue fully a symptom and its history.

(6) Understand, be sensitive and use non-verbal con munication.

(7) Interrupt the patient without disturbing the essentia quality and systematic flow of the interview.

(8) Deploy open-ended questions and closed questions appropriately in pursuit of precision, clarification of inconsistencies and a comprehensive understanding of the problem.

(9) Tolerate emotionally disturbing things which the patient might say or otherwise do.

(10) Use a style that is appropriate to each patient at each stage of the interview.

(11) Avoid jargon and explain the meaning of medical termsu

(12) Define and communicate, when appropriate, underlying motives and meanings of the consultation that reveap themselves as it proceeds.

(13) Recognize denial and other defensiveness and minimize it by one's manner and by interpretation or challenge when appropriate.

(14) Recognize that an interview may be failing in its purpose and make appropriate adjustments.

(15) Answer questions clearly and accurately.

(16) Remember what has been said. 
(17) Help the patient to understand one's recommendations and the effects of accepting or rejecting them.

(18) Formulate and summarize well for the record.

Many of the foregoing abilities in communication with patients also apply to communication between the doctor and other health care professionals. Good communication at this level must be based on an understanding of the respective roles of professional colleagues (such as general practitioners and hospital specialists, and other health care workers in a clinical team) and the appreciation of the contribution which each makes to patient care, to primary prevention and health education management. Goals of such training should be to instil understanding of these factors and of the communication and decision-making processes within the health care system (such as committee structures).

\section{Content of teaching}

\section{A. Relating to the patient}

\section{Knowledge}

The trainee should learn about:

(a) The nature of the relationship between proper professional and optimal therapeutic engagement with patients.

(b) The physical settings that might promote or impair the establishment of a working relationship, e.g. levels of noise, comfort, interruptions.

(c) Aspects of the trainee himself that might promote or impair the establishment of a working relationship (e.g. appearance, manner, demeanour).

(d) Techniques that facilitate the expression of complaint or symptom, e.g. use of non-verbal communication - gesture, affirmatory noise, verbal communications as open and closed questions, empathic reflections.

(e) The importance of the end phase of interviewing and its associated practical problems in the maintenance of a working relationship with the patient.

(f) The concept of 'the patient'; recognizing the potential within it for meeting excessive and self-perpetuating dependency needs on the one hand and, in contrast, the potential that the doctor has to optimize the patient's decision that he has something 'wrong' with himself, of which he wishes to be rid, with the help of the doctor.

\section{Skills}

The trainee should practise:

(a) Introducing himself to the patient indicating the purpose of any interview and in a manner which will maximize the likelihood of engaging the patient in a working relationship.

(b) Empathy, including within those circumstances where there are major ethnic or cultural differences (e.g. a patient or a doctor whose first language is not English), major age discrepancies or discrepancies in intelligence (e.g. a patient with mental handicap or dementia).

(c) Conducting a clinical interview which enables the patient to volunteer personal and other relevant information and to feel involved in his own treatment.

(d) Terminating an interview, at a point which is considered appropriate, without impairing the working relationship (if this is, for therapeutic purposes, to be maintained).

\section{Attitudes}

The trainee should practise: attentiveness and receptivity, being non-judgemental, warmth or detached concern as appropriate, and firm, uncompromising or confronting stances, if appropriate.

\section{B. Eliciting information relevant to history-taking and mental state examination}

\section{Knowledge}

The trainee should learn about:

(a) Conventional headings and categories which make up the case history-taking process.

(b) Conventional headings and categories which make up mental state examination.

(c) Importance of a patient's own attitude to his condition (including insight, consent to treatment).

(d) Importance of a patient's psychological defensive strategies and their possible distorting effect on imparting information to a doctor. This includes an awareness that the most relevant information is often the most difficult for the patient to recall and yield. It also includes an understanding of the impact of the doctor's image/role on the doctor/patient relationship together with its transference aspects.

(e) The nature of non-verbal communication.

II. Skills

The trainee should practise:

(a) Pursuing a complaint and its history and the psychological, personal and social aspects of that history.

(b) Eliciting abnormalities of the mental state (including neurotic and psychotic phenomena).

(c) Tolerating emotionally disturbing affects evoked by a patient's manner or present history.

(d) Conducting a clinical interview with reluctant or 'difficult' patients (e.g. alcoholic, drug-dependent, dysmorphophobic, HIV-positive, non-compliant diabetic, psychopathic, psychotic, suicidal).

(d) Assessing the patient's attitude to his condition (e.g. insight, consent to treatment).

\section{Attitudes}

The trainee should learn to show the same attitudes as listed above and thoroughness.

\section{Integrating information from history and mental state examination}

\section{Knowledge}

The trainee should learn about:

(a) Details of conventional 'formulation' of clinical problems.

(b) Concepts which recognize complex aetiology of many syndromes in terms of predisposition, percipitation and perpetuation.

(c) Aetiological concepts which recognize the interaction of soma and psyche. 


\section{Skills}

The trainee should practise a methodological approach to history-taking, using testable hypotheses, to enable an aetiological statement by the end of a clinical interview (especially initial consultation).

\section{Attitudes}

The trainee should learn to show attitudes as listed previously and also focus on objectivity.

\section{Imparting information to patients and/or colleagues.}

With regard to knowledge the trainee should learn about: (a) The importance of clear communication to patients regarding diagnosis and/or treatment, appropriate to an individual patient's needs, to enable their meaningful, active participation in any treatment which is planned, and (b) The importance of clear communication with professional colleagues (especially in multidisciplinary team settings or in circumstances of multi-agency involvement in treatment or management).

For the necessary skills the trainee should practise imparting information to patients and/or colleagues in order to further the goal of successful treatment via appropriate active participation of the patient and/or the co-operative teamwork of the professionals.

The trainee should acquire and display the attitudes as listed above $(\mathrm{A}-\mathrm{C})$.

\section{Educational process}

Doctors entering postgraduate training may already have well-developed clinical communication skills. The principal way that trainees learn is through their apprenticeship role within the day to day clinical setting. Communications, between trainee and patient, trainee and consultant, are largely inculcated within such related settings as out-patient clinics, ward rounds and case presentations. The consultant trainer should emphasize the importance of a high level of such skills through his own practice and through specific teaching. This can be complemented by specific teaching seminars by clinical tutors.

It can be further augmented within courses, e.g. 12 half day-long workshops focussing on the acquisition of different clinical communication skills (including the skills involved in professional clinical examinations) utilizing seminar, roleplay, videotape, 'homework' and some didactic methods as well as involvement in an expertly conducted sensitivity group. Such training will need to be reinforced at intervals thereafter throughout professional life within the framework of continuing medical education.

Acquisition of the necessary knowledge, skills and attitudes in counselling and psychotherapy practices in medicine requires additional training. Some suggested goals and objectives in this respect, together with a statement of the necessary content, processes and governance have also been outlined elsewhere. ${ }^{5}$
Annexe 2

Additional goals for doctors engaging in communication acros ethnic boundaries

1. Ability to undertake such training and to maintain sucle skills which may be especially uncomfortable because they? may require questioning of some of one's basic assump? tions.

2. Knowledge of the patient's cultural background, botk historical and current, and an understanding of the specia品 and differing problems of first and subsequent generation immigrants to the country.

3. Particular knowledge concerning the cultural influence on $\vec{p}$ health and disease and on attitudes to health and disease in $\vec{L}$ the patient's cultural background.

4. Understanding by the doctor of his attitudes to the patient in terms of his own culture, both historical and current $\frac{0}{3}$ and, relatedly, his attitude to the patient's background taking into account his own immigrant background aṣ relevant.

5. Ability to modify such attitudes as part of his acquisition of good communication skills, as outlined in Annexe 1.ज़

\section{Educational content and processes}

Such abilities can be acquired without excessive commitmen of additional further teaching time though some specific initial and continuing training is necessary. Much inform? ation can be acquired through the written word and gheo informed audio visual media such as public televisian ${ }^{\circ}$ However, expert supervision permitting, the testing outof such acquired ideas is necessary. Supervision can only come from those already expert in such communication skills.

Supervised practical experience should include the team work skills which involve an interpreter under appropriate conditions.

Experience with a mixed culture sensitivity group, that meets at least fortnightly over a period of six months, should 9 comprise the main basis of the initial formal training? Preliminary experience of such training should occur at undergraduate level but the course itself is probably besi undergone shortly after graduation and before or during basic specialist training. Periodic involvement, say sixmonthly, in such groups should arise thereafter throughou․ professional life as part of the further development and maintenance of medical practitioner communication skills ing general.

Though such training is demanding for doctors - just one more educational demand some might say - the authors believe that it effectively addresses an inescapable matter andE. that, put into practice, it would not only sometimes helps patients but also make practice more enjoyable and cost effective. 


\section{References}

1. Education Committee, General Medical Council. Recommendations on General Clinical Training. October 1987.

2. Education Committee, General Medical Council. Recommendations on the Training of Specialists - issued in pursuance of section 15 of the Medical Act 1978. October 1987.

3. Balint, P. The Doctor, the Patient and His Illness. Pitman Medical, London, 1964.

4. Nuffield Provincial Hospitals Trust. Talking with patients. A teaching approach. London. 1985.

5. Crisp, A.H. Undergraduate training for communication in medical practice. $J R$ Soc Med 1986, 79: 568-574.

6. Education Committee, General Medical Council. The Teaching of Behavioural Sciences, Community Medicine and General Practice in Basic Medical Education. March 1987.

7. Heavey, A.P. Learning to talk with patients. Br J Hosp Med 1988, May: 433-439.

8. Mares, P., Henley, A. \& Baxter, C. Health Care in Multi-Racial Britain. Health Education Council and National Extension College Trust, Cambridge, 1985.
9. Coombe, V. \& Little, A. Race and Social Work - A Guide to Training. Tavistock, London, 1986.

10. Education Committee, General Medical Council. Recommendations on Basic Medical Education - issued in pursuance of section 15 of the Medical Act 1978. February 1980.

11. Advisory Committee on Medical Training, Commission of European Communities. Medical Training in the European Community. Proceedings of a Conference held in Brussels 18/19 June 1985 by the Advisory Committee on Medical Training of the Commission of the European Communities. Springer-Verlag, Berlin, 1987.

12. Crisp, A.H., Burns, T., Drummond, L. et al. The Learning of Communication Skills and Psychotherapy by Doctors training in Psychiatry and preparing for entry into Higher Specialist Training. Report of a Working Party accepted for implementation by the South West Thames Regional Postgraduate Medical Committee, 40 Eastbourne Terrace, London W2 3QR. May 1987. 